\title{
Farmacocinética comparativa de una dosis única de ampicilina en llamas luego de la administración intravenosa, intramuscular y subcutánea\#
}

\author{
Comparative pharmacokinetics of a single dose of ampicillin following intravenous, intramuscular \\ and subcutaneous administration to llamas
}

\author{
V Kreil ${ }^{a *}$, AP Prados ${ }^{\mathrm{a}}$, L Ambros ${ }^{\mathrm{a}}$, A Monfrinotti ${ }^{\mathrm{a}}$, P Quaine ${ }^{\mathrm{a}}$, R Hallua ${ }^{\mathrm{a}}$ M Rebuelto ${ }^{\mathrm{a}}$ \\ ${ }^{a}$ Cátedra de Farmacología, Facultad de Ciencias Veterinarias, Universidad de Buenos Aires, Argentina.
}

\begin{abstract}
SUMMARY
Six healthy, adult llamas (Lama glama) received a single intravenous (i.v.), intramuscular (i.m.) and subcutaneous (s.c.) administration of ampicillin at a dosage of $20.0 \mathrm{mg} / \mathrm{kg}$ body weight. Venous blood samples were collected, and ampicillin plasma concentrations were determined by microbiological assay. Kinetic calculations were done using noncompartmental analysis. The period of time that plasma concentrations spent above the minimum inhibitory concentration (T > MIC) was graphically determined. Following the i.v. administration, mean pharmacokinetic parameters $( \pm$ SD) were as follows: terminal elimination half-life $\left(\mathrm{t}_{\mathrm{t} / 2 \mathrm{2}}\right) 0.52 \pm 0.1 \mathrm{~h}$, total body clearance $\left(\mathrm{Cl}_{\mathrm{t}}\right) 10.3 \pm 1.3 \mathrm{ml} / \mathrm{kg} \cdot \mathrm{min}$, volume of distribution at steady state $\left(\mathrm{V}_{\mathrm{ss}}\right)$ $0.35 \pm 0.07 \mathrm{l} / \mathrm{kg}$. Following the i.m. and the s.c. administration, significant differences were determined for peak concentrations $(34.1 \pm 6.2$ and $18.6 \pm$ $6.3 \mu \mathrm{g} / \mathrm{ml}$, respectively), time to peak concentrations $\left(0.26 \pm 0.08\right.$ and $0.6 \pm 0.2 \mathrm{~h}$, respectively); $\mathrm{t}_{1 / 2 \lambda}(0.83 \pm 0.17$ and $1.66 \pm 0.6 \mathrm{~h}$, respectively); mean residence time $(1.3 \pm 0.3$ and $2.7 \pm 1.0 \mathrm{~h}$, respectively), and mean absorption time $(0.72 \pm 0.2$ and $2.1 \pm 0.9 \mathrm{~h}$, respectively). T $>$ MIC values suggest that different ampicillin s.c. and i.m. dosing should be used in llamas, as s.c. injection provides therapeutically effective plasma concentrations for a longer duration.
\end{abstract}

Key words: camelids, pharmacokinetics, antibiotics, ampicillin.

\section{RESUMEN}

Los objetivos de este estudio fueron describir y comparar la farmacocinética y biodisponibilidad de la ampicilina administrada por las vías intravenosa (i.v.), intramuscular (i.m.) y subcutánea (s.c.) a llamas (Lama glama) adultas sanas, y calcular los correspondientes tiempos sobre la CIM (T > CIM) a fin de proveer una base farmacológica para la terapia antimicrobiana racional en esta especie. Seis llamas recibieron una dosis de ampicilina por las vías i.v., i.m. y s.c. Se extrajo sangre a tiempos predeterminados y se calculó las concentraciones plasmáticas utilizando el método microbiológico. Los parámetros farmacocinéticos se calcularon de las curvas de disposición correspondientes. El T > CIM se calculó gráficamente. Los parámetros medios luego de la administración i.v. ( \pm DE) fueron: vida media de eliminación $\left(\mathrm{t}_{1 / 2}\right) 0,52 \pm 0,1 \mathrm{~h}$, clearance $\left(\mathrm{Cl}_{\mathrm{t}}\right) 10,3 \pm 1,3 \mathrm{ml} / \mathrm{kg} \cdot \mathrm{min}$, volumen de distribución al estado estacionario $\left(\mathrm{V}_{\mathrm{ss}}\right) 0,35 \pm 0,07 \mathrm{l} / \mathrm{kg}$. Luego de las administraciones i.m. y s.c., se encontraron diferencias significativas para las concentraciones máximas (34,1 6,2 y 18,6 $\pm 6,3 \mu \mathrm{g} / \mathrm{ml}$, respectivamente), tiempo a la concentración máxima $\left(0,26 \pm 0,08\right.$ y $06 \pm 0,2 \mathrm{~h}$, respectivamente); $\mathrm{t}_{1 / 2 \lambda}(0,83 \pm$ 0,17 y $1,66 \pm 0,6 \mathrm{~h}$, respectivamente); tiempo medio de residencia ( $1,3 \pm 0,3$ y $2,7 \pm 1,0 \mathrm{~h}$, respectivamente), y de absorción $(0,72 \pm 0,2$ y $2,1 \pm 0,9 \mathrm{~h}$, respectivamente). Los valores de T > CIM sugieren que en llamas deberían usarse diferentes dosificaciones cuando se administra la ampicilina por vía i.m. o s.c., ya que la inyección s.c. provee concentraciones plasmáticas terapéuticamente efectivas por más tiempo.

Palabras clave: camélidos, farmacocinética, antibióticos, ampicilina.

\section{INTRODUCCIÓN}

Los camélidos sudamericanos (CS) como las llamas (Lama glama) y alpacas (Vicugna pacos), se crían en la actualidad para la producción de lana y carne, en sistemas intensivos. La alta densidad de animales que existe en estos sistemas aumenta la susceptibilidad de los mismos

Aceptado: 14.11.2013.

\# Proyecto 20020100100698, UBACYT 2011-2014, Secretaría de Ciencia y Técnica, Universidad de Buenos Aires, Argentina.

* Chorroarín 280 (1427), Ciudad Autónoma de Buenos Aires, Argentina; kreil@fvet.uba.ar a los agentes infecciosos. Escherichia coli, Clostridium perfringens, Salmonella, Streptococcus equi sp zooepidemicus y Corynebacterium pseudotuberculosis pueden producir infecciones en llamas y alpacas (Anderson y col 2004, Jones y Boileau 2009). Para diseñar una terapéutica antimicrobiana racional, se deben conocer las características farmacocinéticas propias de las drogas en la especie a tratar, ya que no siempre es adecuado extrapolar de una especie a la otra. En algunas situaciones, los camélidos se tratan aplicando los esquemas posológicos destinados a los rumiantes. Sin embargo, esta extrapolación puede ser errónea ya que existen diferencias anatómicas y fisiológicas entre especies (Lashev y Pashov 1992). La falta 
de datos farmacocinéticos para el cálculo de dosis de los antibióticos puede llevar a fracasos terapéuticos y a la aparición de efectos adversos, incluida la emergencia de cepas resistentes al antibiótico. Si la carne será destinada a consumo, la falta de conocimiento de la fase de eliminación impide el cálculo de tiempos de retirada. Hasta la fecha, son pocos los antibióticos que están aprobados para ser utilizados en CS, careciéndose así de posologías específicas de la especie y de tiempos de retiro válidos.

La ampicilina es un antibiótico beta-lactámico, frecuentemente utilizado en medicina veterinaria por su actividad bactericida, escasa toxicidad y bajo costo. Es activo contra muchas bacterias Gram-negativas, como Escherichia coli, Proteus mirabilis y Salmonella, Borrelia sp, Haemophilus sp, Pasteurella sp, y sobre algunos cocos Grampositivos como estreptococos y estafilococos; es también activa sobre algunos anaerobios como Clostridium sp. No son sensibles Klebsiella sp. y Pseudomonas aeruginosa (Prescott, 2006). La actividad de la ampicilina es tiempodependiente, esto es, la muerte de las bacterias está estrechamente vinculada a la concentración de la droga y a la duración de la exposición de la bacteria a ésta (Craig 1998, Drusano 2004). El índice farmacocinético/farmacodinámico que predice con más exactitud la eficacia clínica de los antibióticos tiempo-dependientes es el tiempo durante el cual las concentraciones plasmáticas permanecen por arriba de la concentración inhibitoria mínima (CIM) para el patógeno causal ( $\mathrm{T}>\mathrm{CIM}$ ) (Toutain y col 2002).

La farmacocinética de la ampicilina ha sido previamente descripta en camellos (Al Nazawi, 2003), alpacas (Kreil y col 2001), y llamas (Christensen y col 1996) luego de su administración por las vías intravenosa (i.v.) e intramuscular (i.m.). En la actualidad, las formulaciones de ampicilina soluble están indicadas para la inyección i.v., i.m y subcutánea (s.c.). En la práctica clínica, se considera que la disposición de las drogas es similar cuando se utilizan las vías i.m. y s.c. Sin embargo, hemos probado previamente que la vía de administración puede alterar la absorción de las drogas (Waxman Dova y col 2008), pudiendo verse comprometido tanto el éxito del tratamiento como la salud del consumidor.

Los objetivos de este estudio fueron describir y comparar la farmacocinética y biodisponibilidad de la ampicilina administrada por las vías i.v., i.m. y s.c. a llamas adultas sanas, y calcular los correspondientes T > CIM, utilizando valores de bibliografía, a fin de proveer una base farmacológica para la terapia antimicrobiana racional en esta especie.

\section{MATERIAL Y MÉTODOS}

\section{ANIMALES}

Para esta experiencia se utilizaron seis llamas adultas, de 4 a 7 años de edad, de peso (media \pm DE) 117,0 $\pm 15,5 \mathrm{~kg}$. Para el cálculo de la dosis a administrar, cada llama se pesó el día del tratamiento. Los animales estaban clínicamente sanos según examen físico y hematológico. Todas las llamas permanecieron estabuladas en un galpón durante la experiencia, con acceso ad libitum a alimentación verde y agua. El protocolo de investigación fue aprobado por el Comité Institucional de Cuidado y Uso de los Animales de Laboratorio de la Facultad de Ciencias Veterinarias, Universidad de Buenos Aires.

\section{DISEÑO EXPERIMENTAL}

Cada animal recibió $20 \mathrm{mg} / \mathrm{kg}$ de ampicilina sódica por las vías i.v. (vena yugular derecha), i.m. (músculo semitendinoso) y s.c. (debajo de un pliegue de la piel sobre el lateral del tórax), siguiendo un diseño cruzado randomizado, con un período de lavado de 2 semanas entre tratamientos. Para todos los tratamientos se utilizó una solución acuosa de ampicilina sódica ${ }^{1}$. Se tomaron muestras de sangre $(2 \mathrm{ml})$ de la vena yugular izquierda en tubos heparinizados a las $0,08,0,16,0,33,0,5,0,75,1$, $1,5,2,3,4,5,6$, (i.v.) 8, 10, 12 y 24 (i.m., s.c.) h después de administrada la droga. Las muestras se mantuvieron refrigeradas hasta su centrifugación a $3000 \mathrm{~g}$ por $10 \mathrm{~min}$. El plasma sobrenadante fue refrigerado a $4^{\circ} \mathrm{C}$ hasta su análisis dentro de los 4 días post extracción.

\section{DETERMINACIÓN DE LAS CONCENTRACIONES DE AMPICILINA}

Las concentraciones de ampicilina en plasma se midieron para cada muestra en triplicado, mediante el método microbiológico (Bennett y col 1966), utilizando Bacillus subtilis ATCC 6633 como organismo patrón. El método se consideró apropiado, ya que la ampicilina carece de metabolitos con actividad microbiológica (Papich y Riviere 2009). La curva estándar se preparó en plasma de llamas blanco, sembrándolo simultáneamente con las muestras incógnito. El límite de cuantificación del método fue de $0,39 \mathrm{ug} / \mathrm{ml}$. El coeficiente de correlación de la regresión linear de la curva estándar fue de 0,98 . Los coeficientes de variación intra e inter-día, y la exactitud (sesgo) fueron $<7 \%,<10 \% \mathrm{y}<10 \%$, respectivamente, en el rango de las concentraciones empleadas (100-5025-12,5-6,25-3,125-1,56-0,78-0,39 ug/ml). El límite de cuantificación preciso y exacto fue la menor concentración utilizada para el análisis farmacocinético.

\section{ANÁLISIS FARMACOCINÉTICO}

Los parámetros farmacocinéticos se calcularon de las curvas concentración plasmática en función del tiempo, utilizando un programa computarizado ${ }^{2}$ y realizando un

BioAmpi, Laboratorios BioAmer, Buenos Aires, Argentina, 200 $\mathrm{mg} / \mathrm{ml}$

2 PKSolution, Summit Research Services, USA 
análisis no compartimental. La constante de eliminación (l) se obtuvo de la pendiente de la línea de regresión obtenida de los 4-5 datos de la fase terminal, y la vida media de eliminación $\left(\mathrm{t}_{1 / 2}\right)$ se calculó como 0,693/l. El tiempo medio de residencia (TMR) se calculó según la ecuación $\mathrm{TMR}=\mathrm{ABMC}_{0-\not} / \mathrm{ABC}_{0-\frac{*}{*}}$, donde $\mathrm{ABC}_{0-*}$ es el área bajo la curva de la concentración plasmática en función del tiempo, y $\mathrm{ABMC}_{0-\neq}$ el área bajo la curva de la concentración plasmática multiplicada por el tiempo en función del tiempo, calculadas según el método de los trapezoides y extrapoladas al infinito. El tiempo medio de absorción (TMA) se calculó según TMA $=\mathrm{TMR}_{\mathrm{no} \mathrm{iv}}-\mathrm{TMR}_{\mathrm{iv}}$. El volumen de distribución al estado estacionario $\left(\mathrm{V}_{\mathrm{dss}}\right)$ se calculó como $\mathrm{V}_{\text {dss }}=\mathrm{TMR} \times \mathrm{Cl}_{\mathrm{t}}$, siendo clearance $\left(\mathrm{Cl}_{\mathrm{t}}\right)=$ Dosis/ABC $\mathrm{AB}_{0:-} \mathrm{C}_{\max }$ y el tiempo en alcanzar $\mathrm{C}_{\max }\left(\mathrm{t}_{\max }\right)$ se calcularon gráficamente. La biodisponibilidad (f) se calculó según $\mathrm{ABC}_{\text {no iv }} / \mathrm{ABC}_{\mathrm{iv}}$. Los resultados se expresan como media \pm desviación estándar (DE). El T $>\mathrm{CIM}_{90}$ fue calculado gráficamente para las tres vías de administración para los valores de CIM $_{90}$ reportados en veterinaria, de susceptibilidad moderada $(4 \mu \mathrm{g} / \mathrm{ml})$, descripta para Escherichia coli, Proteus mirabilis y Salmonella, y alta $(0,5 \mu \mathrm{g} / \mathrm{ml})$, descripta para Staphylococcus aureus, Leptospira sp, Actinobacillus sp., Moraxella sp y Pasteurella sp (Prescott 2006). Los valores se expresan en media (intervalo de confianza 95\%) en horas.

\section{ANÁLISIS ESTADÍSTICO}

Para calcular las diferencias entre parámetros calculados luego de los tres tratamientos se utilizó un programa computarizado ${ }^{3}$. Para comparar los valores de $\mathrm{C}_{\max } \mathrm{y}$ ABC se utilizó la transformación logarítmica y el test $T$ de Student o ANOVA respectivamente. Las comparaciones entre los tres grupos se realizaron mediante ANOVA de medidas repetidas, utilizando el post test de comparación múltiple de Tukey cuando se detectasen diferencias significativas. La comparación entre $t_{\max }$, TMA, f y T > CIM luego de las administraciones i.m. y s.c. se realizaron mediante el test de Wilcoxon. El nivel de significación fue $\mathrm{P}<0,05$ (Lacey y col 1991).

\section{RESULTADOS}

La ampicilina fue bien tolerada por las llamas, y no se observaron reacciones adversas. Las curvas de concentraciones plasmáticas (media $\pm \mathrm{EE}$ ) versus tiempo obtenidas para los tres tratamientos se muestran en la figura 1. Los parámetros farmacocinéticos calculados para las tres vías se muestran en la cuadro 1 .

Los resultados muestran que la ampicilina se absorbió en forma similar por las vías i.m. y s.c. como lo demuestra la falta de diferencias entre $\mathrm{ABC}_{0-\infty}$ y la biodisponibi-

\footnotetext{
GraphPad Prism, 5.0, GraphPad Software Inc, San Diego, California, USA
}

lidad absoluta. Sin embargo, se observaron diferencias entre algunos parámetros. $\mathrm{La} \mathrm{C}_{\max }$ fue significativamente más alta y el $t_{\max }$, TMR, TMA y $t_{1 / 2 \lambda}$ fueron significativamente más cortos después de la administración i.m. que luego de la s.c. (cuadro 1).

Los valores de $\mathrm{T}>\mathrm{CIM}_{90}$ calculados luego de las tres administraciones se muestran en la cuadro 2 . Las rutas i.m. y s.c. mostraron valores significativamente diferentes para los patógenos de moderada $(4 \mu \mathrm{g} / \mathrm{ml})$ y alta $(0,5$ $\mathrm{mg} / \mathrm{ml}$ ) susceptibilidad.

\section{DISCUSIÓN}

La ampicilina es un antibiótico beta-lactámico con buena actividad contra numerosos patógenos de frecuente aparición en infecciones en camélidos (Anderson y col 2004) y se ha indicado su uso en llamas por las vías i.v. e i.m. (Anderson y col 2004, Jones y Boileau 2009). En este estudio hemos descrito el comportamiento farmacocinético de la ampicilina luego de su administración en llamas por las vías parenterales más comúnmente utilizadas en la práctica clínica. Nuestras condiciones de ensayo mostraron que el perfil farmacocinético y terapéutico de la ampicilina es significativamente diferente cuando se aplica por la vía i.m. o s.c., por ello estas inyecciones no deben ser consideradas intercambiables.

La ampicilina se absorbió más rápidamente por la vía i.m. en comparación a la s.c., según lo mostrado por una $\mathrm{C}_{\max }$ significativamente más alta, $\mathrm{y} \mathrm{t}_{\max }$ y TMA significativamente más cortos que los calculados luego de la administración s.c. La eliminación también mostró diferencias, evidenciadas por una $t_{1 / 2 \lambda}$ y TMR más cortos luego de la administración i.m. Estas diferencias en los parámetros farmacocinéticos eran esperadas, y se deberían muy probablemente a la absorción más lenta que tiene lugar cuando se administra el preparado por vía s.c. Esta absorción extendida puede afectar la tasa de eliminación de la droga (fenómeno de flip-flop), causando la prolongada vida media observada. La biodisponibilidad absoluta, sin embargo, fue similar después de los dos tratamientos, indicando que el grado de absorción de la ampicilina fue similar luego de las dos administraciones.

Se han publicado escasos estudios describiendo la farmacocinética de la ampicilina en CS, y según nuestro conocimiento, ninguno de ellos describe la administración s.c., aún cuando esta ruta es frecuentemente usada en la práctica clínica. Luego de la administración i.v., la $\mathrm{t}_{1 / 2 \lambda}$ y el $\mathrm{Cl}_{\mathrm{t}}$ en este estudio son similares a aquellos reportados en alpacas $(0.6 \pm 0.2 \mathrm{~h} \mathrm{y} 16 \pm 3 \mathrm{ml} / \mathrm{min} \cdot \mathrm{kg}$, respectivamente) (Kreil y col 2001). Sin embargo, un estudio previo en llamas indicó un $\mathrm{Cl}_{\mathrm{t}}$ similar al nuestro $(0,88 \pm 0,16 \mathrm{ml} / \mathrm{min} \cdot \mathrm{kg})$, pero una $\mathrm{t}_{1 / 2 \lambda}$ más prolongada $(3,3 \pm 0.5$ h) (Christensen y col 1996). Nuestro TMR fue similar al descripto previamente en alpacas $(0,7 \pm 0,1 \mathrm{~h})$ (Kreil y col 2001) pero diferente al descripto en llamas $(5,0 \pm 0,6$ h) (Christensen y col 1996). Estas diferencias 


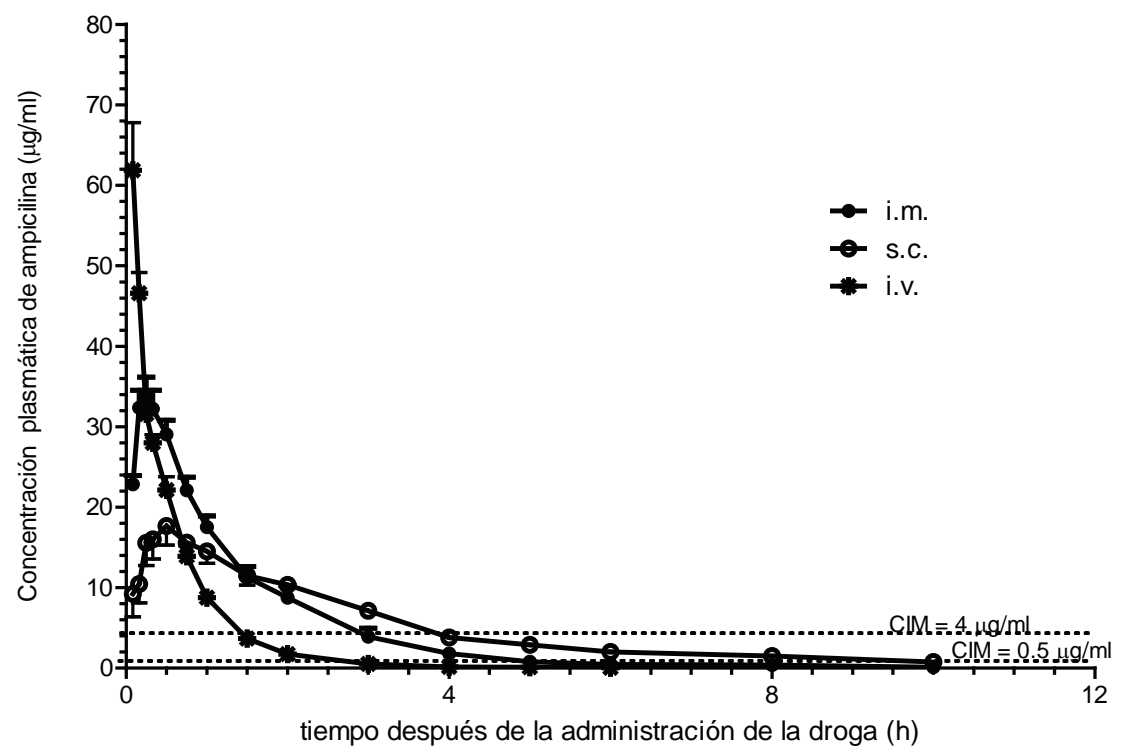

Figura 1. Curva de las concentraciones plasmáticas (media \pm EE) de ampicilina sódica versus el tiempo, luego de la administración de una dosis única de $20 \mathrm{mg} / \mathrm{kg}$ por la vía intravenosa (i.v), intramuscular (i.m.) y subcutánea (s.c.) a 6 llamas adultas.

Plasma concentration vs time curve after intravenous (i.v.), intramuscular (i.m.) and subcutaneous (s.c.) injection of $20 \mathrm{mg} / \mathrm{kg}$ sodium ampicillin to six adult llamas (mean $\pm \mathrm{SE}$ ).

Cuadro 1. Parámetros farmacocinéticos (media \pm DE) de la ampicilina (20 mg/kg) luego de su administración intravenosa (i.v.), intramuscular (i.m.) y subcutánea (s.c.) a 6 llamas.

Pharmacokinetic parameters (mean $\pm \mathrm{SD}$ ) of ampicillin after intravenous (i.v.), intramuscular (i.m.) and subcutaneous (s.c.) administration of sodium ampicillin $(20 \mathrm{mg} / \mathrm{kg})$ to 6 llamas.

\begin{tabular}{lccc}
\hline \multirow{2}{*}{ Parámetro } & \multicolumn{3}{c}{ Vía de administración } \\
\cline { 2 - 4 } & IV & IM & SC \\
\hline $\mathrm{C}_{\text {max }}(\mu \mathrm{g} / \mathrm{ml})$ & - & $34.1 \pm 6.2^{\mathrm{a}}$ & $18.6 \pm 6.3^{\mathrm{b}}$ \\
$\mathrm{t}_{\text {max }}(\mathrm{h})$ & - & $0.26 \pm 0.08^{\mathrm{a}}$ & $0.6 \pm 0.2^{\mathrm{b}}$ \\
$\lambda\left(\mathrm{h}^{-1}\right)$ & $1.4 \pm 0.2$ & $0.86 \pm 0.16$ & $0.47 \pm 0.17$ \\
$\mathrm{t}_{1 / 2 \lambda}(\mathrm{h})$ & $0.52 \pm 0.1^{\mathrm{a}}$ & $0.83 \pm 0.17^{\mathrm{a}}$ & $1.66 \pm 0.6^{\mathrm{b}}$ \\
$\mathrm{ABC}_{0-\mathrm{n}}(\mu \mathrm{g} \cdot \mathrm{h} / \mathrm{ml})$ & $28.0 \pm 4.4^{\mathrm{a}}$ & $48.0 \pm 13.3^{\mathrm{b}}$ & $50.3 \pm 6.8^{\mathrm{b}}$ \\
$\mathrm{ABC}_{0-\infty}(\mu \mathrm{g} \cdot \mathrm{h} / \mathrm{ml})$ & $33.0 \pm 4.7^{\mathrm{a}}$ & $49.1 \pm 13.1^{\mathrm{b}}$ & $52.4 \pm 7.2^{\mathrm{b}}$ \\
$\mathrm{TMR}_{\mathrm{a}}(\mathrm{h})$ & $0.57 \pm 0.1^{\mathrm{a}}$ & $1.3 \pm 0.3^{\mathrm{a}}$ & $2.7 \pm 1.0^{\mathrm{b}}$ \\
$\mathrm{TMA}(\mathrm{h})$ & - & $0.72 \pm 0.2^{\mathrm{a}}$ & $2.1 \pm 0.9^{\mathrm{b}}$ \\
$\mathrm{Cl}_{\mathrm{t}}(\mathrm{ml} / \mathrm{min} \cdot \mathrm{kg})$ & $10.3 \pm 1.3$ & - & - \\
$\mathrm{V}_{\mathrm{ss}}(1 / \mathrm{kg})$ & $0.350 \pm 0.07$ & - & - \\
$\mathrm{V}_{\mathrm{z}}(\mathrm{l} / \mathrm{kg})$ & $0.455 \pm 0.07$ & - & - \\
$\mathrm{f}$ & - & $1.5 \pm 0.2^{\mathrm{a}}$ & $1.6 \pm 0.9^{\mathrm{a}}$ \\
\hline
\end{tabular}

a,b diferentes superíndices indican diferencias significativas $(\mathrm{P}<0,05)$.

$\lambda$ : constante de eliminación; $\mathrm{t}_{1 / 2 \lambda}$ : vida media de eliminación; $\mathrm{ABC}_{0-\mathrm{n}}$ : área bajo la curva hasta la última concentración calculada, $\mathrm{ABC}_{0-\infty}$ : área bajo la curva extrapolada hasta infinito; TMR: tiempo medio de residencia; TMA: tiempo medio de absorción; $\mathrm{Cl}_{\mathrm{t}}$ : clearance total; $\mathrm{V}_{\mathrm{dss}}$ : volumen de distribución al estado estacionario; $\mathrm{V}_{\mathrm{z}}$ : volumen aparente de distribución; $\mathrm{C}_{\max }$ : concentración máxima; $\mathrm{T}_{\max }$ : tiempo en llegar a la concentración máxima; f: biodisponibilidad.

$\mathrm{C}_{\max }$ : peak serum concentration; $\mathrm{t}_{\max }$ : time to reach peak serum concentration, $\lambda$ : apparent terminal rate constant; $\mathrm{t}_{1 / 2} \lambda$ : terminal half-life; $\mathrm{ABC}_{0-\mathrm{n}}:$ area under the serum concentration time-curve from time zero to last point; $\mathrm{ABC}_{0-\infty}$ : area under the serum concentration time-curve from time zero to infinity; TMR: mean residence time; TMA: mean absorption time, $\mathrm{Cl}_{\mathrm{t}}$ : total body clearance; $\mathrm{V}_{\mathrm{ss}}$ : volume of distribution at the steady state; $\mathrm{V}_{\mathrm{z}}$ : apparent volume of distribution; f: absolute bioavailability

a,b values within a row with different superscripts indicate significant differences $(\mathrm{P}<0.05)$. 
Cuadro 2. Índice de eficacia clínica farmacocinético/farmacodinámico $\left(\mathrm{T}>\mathrm{CIM}_{90}\right)$ calculado para la ampicilina $(20 \mathrm{mg} / \mathrm{kg})$ considerando bacterias de susceptibilidad moderada (CIM $=4$ $\mu \mathrm{g} / \mathrm{ml})$ y alta $(\mathrm{CIM}=0,5 \mu \mathrm{g} / \mathrm{ml})$ luego de su administración intravenosa (i.v.), intramuscular (i.m.) y subcutánea (s.c.) a 6 llamas.

Efficacy predictor $\left(\mathrm{T}>\mathrm{MIC}_{90}\right)$ calculated for ampicillin $(20 \mathrm{mg} / \mathrm{kg}$ ) against bacteria of moderate (MIC $=4 \mu \mathrm{g} / \mathrm{ml})$ and high (MIC $=0.5 \mu \mathrm{g} / \mathrm{ml}$ ) susceptibility after its intravenous (i.v.), intramuscular (i.m.) and subcutaneous (s.c.) administration to 6 llamas.

\begin{tabular}{cccc}
\hline $\mathrm{CIM}_{90}$ & \multicolumn{3}{c}{$\mathrm{T}>\mathrm{CIM}(\mathrm{h})$} \\
$(\mu \mathrm{g} / \mathrm{ml})$ & \multicolumn{2}{c}{ media (intervalo de confianza 95\%) } \\
\hline & i.v. & i.m. & s.c. \\
4,0 & $1.5(1.3-1.7)$ & $3.0(2.3-3.7)^{\mathrm{a}}$ & $4.3(3.2-5.5)^{\mathrm{b}}$ \\
0,5 & $3.0(2.3-3.7)$ & $5.4(4.2-6.6)^{\mathrm{a}}$ & $8.4(6.1-10.7)^{\mathrm{b}}$ \\
\hline
\end{tabular}

a,b diferentes superíndices indican diferencias significativas $(\mathrm{P}<0,05)$.

${ }^{\mathrm{a}, \mathrm{b}}$ values within a row with different superscripts indicate significant differences $(\mathrm{P}<0.05)$.

pueden ser debidas a factores relacionados a los animales, a la preparación farmacológica y al diferente análisis farmacocinético aplicado por los distintos autores. $\mathrm{El} \mathrm{V}_{\mathrm{ss}}$ medio calculado en nuestro estudio es congruente con la limitada difusión extravascular de los antibióticos betalactámicos, y es más bajo $(0,6 \pm 0,051 / \mathrm{kg})$ (Kreil y col 2001) o similar $(0,277 \pm 0,08 \mathrm{l} / \mathrm{kg})$ (Christensen y col 1996) a aquellos previamente informados para alpacas y llamas, respectivamente. Los valores medios de $t_{1 / 2 \lambda}$ y TMR calculados en este estudio luego de la administración i.m. son similares a los calculados para alpacas $(0,8$ $\pm 0,5$ y $0,9 \pm 0,3 \mathrm{~h}$, respectivamente) (Kreil y col 2001).

Para los antibióticos tiempo-dependientes, el índice farmacocinético/farmacodinámico que mejor puede predecir la probabilidad de erradicación bacteriana y resultado clínico es el T > CIM. Los antibióticos betalactámicos presentan actividad tiempo-dependiente, y su éxito clínico es mayor cuando las concentraciones plasmáticas exceden las CIM contra el patógeno causal durante al menos el 40 - 50\% del intervalo posológico (Toutain 2002). En este estudio hemos utilizado CIM reportadas previamente para microorganismos aislados en especies veterinarias para el cálculo del $\mathrm{T}>\mathrm{CIM}$, ya que no hemos encontrado datos de aislamientos clínicos de CS. Nuestros resultados muestran que para bacterias de susceptibilidad moderada (CIM $=4 \mu \mathrm{g} / \mathrm{ml})$, las concentraciones plasmáticas de la ampicilina se mantienen por arriba de este valor durante al menos 1,0, 2,0 y 3,0 h, después de la inyección i.v., i.m. o s.c., de $20 \mathrm{mg} / \mathrm{kg}$ de ampicilina, respectivamente. Esta duración es más prolongada cuando se considera la CIM de patógenos muy susceptibles $(\mathrm{CIM}=0,5 \mu \mathrm{g} / \mathrm{ml})$, ya que se mantienen concentraciones arriba de la CIM por al menos 2,0, 4,0 y $6,0 \mathrm{~h}$ luego de las inyecciones i.v., i.m. o s.c., respectivamente. Estos resultados sugieren que el régimen poso- lógico debería ser diferente cuando se aplica por vía i.m o s.c., ya que ambas proveen diferente exposición bacteriana. Las dosis de $20 \mathrm{mg} / \mathrm{kg}$ de ampicilina deberían darse por vía i.m. cada 6-8 h (patógenos de sensibilidad moderada, como bacilos entéricos Gram-negativos), o cada $12 \mathrm{~h}$ (patógenos de alta susceptibilidad, como cocos Gram-positivos); mientras que, si se utiliza esta dosificación por vía s.c., los intervalos podrían prolongarse a 8-12 h o 12- 24 h, respectivamente. Si la infección fuese causada por microorganismos de susceptibilidad marginal, cuyas CIM se encuentran próximas al punto de corte de la ampicilina, se debería utilizar una dosificación mayor a la empleada en este estudio. La vía i.v. provee una buena profilaxis con el fin de reducir la incidencia de infecciones en el sitio quirúrgico, cuando se supone contaminación por bacterias susceptibles a la ampicilina, como sucede en las cirugías dentales y abdominales.

En la actualidad, las llamas pueden proveer no solo lana sino también carne. Los tiempos de retiro se recomiendan a fin de proteger la salud del consumidor, y tienen como base para su cálculo la tasa de eliminación de la droga (Concordet y Toutain 1997). Por ello, la diferente permanencia de la ampicilina luego de la administración i.m. o s.c. debe ser considerada cuando se calcula el tiempo de retirada del preparado comercial.

En conclusión, nuestros resultados sugieren que la ampicilina puede ser un agente antimicrobiano eficaz para el tratamiento de infecciones en llamas. La vía s.c. muestra un perfil farmacocinético más ventajoso que la i.m., visto que las concentraciones plasmáticas se mantienen por más tiempo por sobre las CIM de los patógenos frecuentes, lo cual permite administraciones más espaciadas. Además, debido a la pequeña masa muscular de los CS, las inyecciones s.c. son más recomendables que las i.m. Los próximos estudios deberán contemplar la farmacocinética de dosis múltiples de ampicilina, posibles efectos adversos, y susceptibilidad de aislamientos de CS a la ampicilina. Así mismo, si se pretende utilizar la carne para consumo humano, se debe tener en cuenta la vía de administración para el cálculo del período de retirada.

\section{AGRADECIMIENTOS}

Este estudio es parte del Proyecto 20020100100698, UBACYT 2011-2014, Secretaría de Ciencia y Técnica, Universidad de Buenos Aires, Argentina.

\section{REFERENCIAS}

Al Nazawi MH. 2003. Comparative pharmacokinetic studies on ampicillin in camels, sheep and goats. Pak J Biol Sci 6,1005-1008

Anderson DE, DM Rings, J Kowalski. 2004. Infection with Corynebacterium pseudotuberculosis in five alpacas. $J$ Am Vet Med Assoc 225, 1743-1747.

Bennet JV, JL Brodie, EJ Benner, WM Kirby. 1966. Simplified, accurate method for antibiotic assay of clinical spec- 
imens. Appl Microbiol 14, 170-177.

Christensen JM, BB Smith, SB Murdane, N Hollingshead. 1996. The disposition of five therapeutically important antimicrobial agents in llamas. J Vet Pharmacol Ther 19, 431-438.

Concordet D, PL Toutain. 1997. The withdrawal time estimation of veterinary drugs: a non-parametric approach. $J$ Vet Pharmacol Ther 20, 374-379.

Craig WA. 1998. Pharmacokinetic/pharmacodynamic parameters: rationale for antibacterial dosing of mice and men. Clin Infect Dis 26, 1-12.

Drusano GL. 2004. Antimicrobial pharmacodynamics: critical interactions of "bug and drug". Nat Rev Microbiol 2, 289-300.

Jones M, M Boileau. 2009. Camelid Herd Health. Vet Clin Food Anim 25, 239-263.

Kreil V, C Lüders, R Hallu, M Rebuelto, L Betancourt. 2011. Pharmacokinetics of ampicillin in Alpacas (Lama pacos). Arch Med Vet 33, 241-246.

Lacey LF, ON Keene, JF Pritchard, A Bye. 1997. Common non compartmental pharmacokinetic variables: are they normally or log-normally distributed? J Biopharm Stat 7, 171-178.

Lashev LD, DA Pashov. 1992. Interspecies variations in plas- ma half-life of ampicillin, amoxycillin, sulphadimidine and sulphacetamide related to variations in body mass. ResVet Sci 53, 160-164.

Papich MG, JE Riviere. 2009. B-lactam antibiotics: penicillins, cephalosporins and related drugs. In: Riviere JE, Papich MG, Adams HR (eds). Veterinary Pharmacology and Therapeutics. $9^{\text {th }}$ ed. Willey-Blackwell, Ames, USA, Pp 866-888.

Prescott JF. 2006. Beta-lactam Antibiotics: Penam Penicillins. In: Giguère S, Prescott JF, Baggot JD, Walker RD, Dowling PM (eds). Antimicrobial Therapy in Veterinary Medicine. $4^{\text {th }}$ ed. Blackwell Publishing, Ames, Iowa, USA, Pp 121-139.

Toutain PL, JRE Del Castillo, A Bousquet-Mélou. 2002. The pharmacokinetic-pharmacodynamic approach to rational dosage regimen for antibiotics. Res Vet Sci 3, 105-114.

Toutain PL, P Lees. 2004. Integration and modelling of pharmacokinetic and pharmacodynamic data to optimize dosage regimens in veterinary medicine. $J$ Vet Pharmacol Ther 27, 467-77.

Waxman Dova S, G Albarellos, V Kreil, L Montoya, L Ambros, R Hallu, M Rebuelto. 2008. Comparative pharmacokinetics of an injectable cephalexin suspension in beef cattle. Res Vet Sci 85, 570-574. 